\title{
Recent Directions in Remote Engineering and Virtual Instrumentation
}

\author{
doi:10.3991/ijoe.v5s1.1032
}

Tarek Sobh, Jani Pallis, Navarun Gupta

The 6th Remote Engineering and Virtual instrumentation Conference (REV 2009) was held at the University of Bridgeport in Bridgeport, Connecticut, USA during the period of June 22 25,2009 . The conference brought together engineering researchers, educators, and professionals to explore the fundamentals, future, and application of remote engineering in both industry and academia. Participants delivered papers, presented demonstrations, research posters, and shared experiences in virtual engineering. REV'09 drew more than 100 engineers, scientists and educators from around the world. M ost of the participants were from Europe, but many came from Asia, North and South America, the Middle East and as far as Australia. M ore than 60 papers were presented on topics ranging from Telerobotics to Virtual and Remote Labs. Workshops and Tutorials drew widespread interest and exhibitors displayed their products for integrating remote engineering into academia. Poster sessions discussed topics such as Robotic Surgery and Development of Remote Labs in Physics.

The general objective of REV 2009 was to discuss fundamentals, applications and experiences within the field of online engineering, both in industry and academia. The conference presentations and papers addressed several emerging trends in online engineering, remote laboratories, virtual instrumentation and educational applications of remote engineering.

The fields of interest and the presented papers in the conference addressed, among others, topics within the following areas:

- Virtual and remote laboratories

- Remote process visualization and virtual Instrumentation

- Remote control and measurement technologies

- Networking and grid technologies

- Mixed Reality environments for education and training

- Demands in education and training, e-learning, b-learning, m-learning and ODL

- Teleservice and telediagnosis

- Telerobotics and telepresence

- Support of collaborative work in virtual engineering environments

- Teleworking environments

- Telecommunities and their social impact

- Human computer interfaces, usability, reusability, accessibility

- Standards and standardization proposals

- Innovative organizational and educational concepts for remote engineering

This special issue of the International Journal of Online Engineering includes 12 selected papers from the REV 2009 conference (www.rev2009.org ). The topics addressed in the 
papers spanned a variety of theoretical and applied research endeavors within the area of online engineering.

The first paper in this special issue is entitled "Helicopter Laboratory M odel Experiment with Web Access", coauthored by V.M. Cvjetkovic, M. S. M atijevic, M. D. Grujovic and M. Z. Stefanovic. A laboratory helicopter model with two degrees of freedom was developed to teach mechanical engineering students static and dynamic characteristics and controls. Different measurements and experiments can be performed with the laboratory helicopter model both locally and remotely. Remote experiments are performed by using a web-based user interface for controlling the laboratory equipment and an IP (Internet Protocol) camera for observing the movements of the helicopter model.

The second paper in this special issue is entitled "Lessons Taught and Learned from the Operation of the Solar Energy e-learning Laboratory", coauthored by P. C. Eleftheriou and I. M. M ichaelides. The paper discusses the authors' experiences and success with the solar energy e-learning laboratory (solar e-lab) in Cyprus. The system is composed of a pilot solar energy conversion plant which is equipped with all necessary instrumentation, data acquisition, and communication devices needed for remote access, control, data collection and processing. Over a 5-year period, the solar e-lab has been accessed by users from over 500 locations from 79 countries across the globe.

The third paper in this special issue is entitled "Collaborative Learning Based on a MicroWebserver Remote Test Controller", coauthored by J. M. Ferreira, E. Sousa, A. Nafalski, J. Machotka and Z. Nedic. This paper presents a remote test workbench that was developed to support on-line assignments dealing with the IEEE 1149.1 standard test access port and boundary-scan architecture. The remote workbench is based on low-cost remote webserver technology and offers an effective solution for the development of remote test controllers able to implement a wide set of on-line experiments for a M oodle-based digital electronics test course offered to engineering students.

The fourth paper in this special issue is entitled "Remote Laboratory Description Language Based On XM L", coauthored by I. A. Grout and A. C. Rodrigues da Silva. This work was awarded Best Paper of the REV 2009 Conference. This paper presents a language used to describe the structure and capabilities (attributes) of remote, or online, laboratories. The structure of the language is presented and an example is given with reference to the specific case study remote laboratory. The language can be readily extended to describe current laboratory attributes in more detail and to extend the language in order to identify and present new laboratory attributes.

The fifth paper in this special issue is entitled "A Versatile Internet-Accessible Electronics Workbench with Troubleshooting Capabilities", coauthored by H. Soumare, R. Shroff, J. L. Hardison, J. A. del Alamo, V. J. Harward, P. H. Bailey and K. K. DeLong. The paper focuses on recent efforts at the MIT iLab Project to build remote laboratories around the NI-ELVIS 
platform, an all-in-one electronics workbench. Recent efforts are detailed on expanding the capabilities of ELVIS-based iLabs to enable students to test and debug digital and analog circuits. These added capabilities will provide educators and students with great flexibility and significantly enrich the remote laboratory experience.

The sixth paper in this special issue is entitled "M obile Prototyping Platforms for Remote Engineering Applications", coauthored by K. Henke, St. Ostendorff, T. Volkert and A. Mitschele-Thiel. The paper describes a low-cost mobile communication platform as a universal rapid-prototyping system, based on the Quadrocopter concept. These mobile platforms are used to motivate bachelor and master students to study Computer Engineering sciences. The multipurpose mobile prototyping platforms provide students the basics for the application of mobile airborne communications. The robust communication platform allows the execution of multiple missions in a short period of time with minimal setup and organization between the experiments.

The seventh paper in this special issue is entitled "Integrated Circuits Testing: Remote Access to Test Equipment for Labs and Engineering", coauthored by B. Pradarelli, L. Latorre and P. Nouet. This paper addresses the local and remote use of an Integrated Circuits (IC) Automated Test Equipment (ATE) for both educational and engineering purposes. Here, practical information regarding IC testing and network setup for remote access are detailed, together with the associated training program.

The eighth paper in this special issue is entitled "Adapting a Remote Laboratory Architecture to Support Collaboration and Supervision", coauthored by David Lowe, Chris Berry, Steve Murray, and Euan Lindsay. For remote laboratories, most attention has been paid to the development of core infrastructure. There has, however, been recent recognition of the importance of considering how collaboration and supervision can also be supported. This paper discusses a novel approach to the integration of support for multi-user distributed access to a single laboratory instance. The approach retains the benefits of the lightweight client inherent in the underlying architecture.

The ninth paper in this special issue is entitled "Online M OS Capacitor Characterization in LabVIEW Environment", coauthored by S. C. Pandey, A. M aiti, T. K. M aiti and C. K. Maiti. This paper presents an automated evaluation procedure to characterize M OS capacitors involving high-k gate dielectrics. Suitability of LabVIEW environment for online web-based semiconductor device characterization is demonstrated. Implementation of the algorithm for use as a remote internet-based characterization tool, where the client and server communicate with each other via web services, is also shown.

The tenth paper in this special issue is entitled "Fully-LabVIEW Powered Mössbauer Spectrometer", coauthored by J. Pechousek, R. Prochazka, D. Jancik and M. Mashlan. In this paper, virtual instrumentation techniques are applied for the first fully-LabVIEW powered Mössbauer spectrometer. The spectrometer application has to carry out several different tasks such as $\gamma$-ray pulse height analysis, reference velocity signal generation for the motion 
of the radioactive source together with digital proportional-integral-derivative (PID) unit responsible to control the relative precise velocity between the source and the absorber and Mössbauer spectra accumulation.

The eleventh paper in this special issue is entitled "Collaborative Virtual 3D Environment for Internet-accessible Physics Experiments", coauthored by Tina Scheucher, Philip H. Bailey, Christian Gutl, and V. Judson Harward. This paper focuses on the integration of internetaccessible physics experiments (iLabs) combined with the TEALsim 3D simulation toolkit in Project Wonderland and Sun's toolkit for creating collaborative 3D virtual worlds. Within such a collaborative environment these tools provide the opportunity for teachers and students to work together as avatars as they control actual equipment, visualize physical phenomenon generated by the experiment, and discuss the results. In particular this paper outlines the steps of integration, future goals, as well as the value of a collaboration space in Wonderland's virtual world.

The twelfth and final paper in this special issue is entitled "Web Development Techniques and

Remote Laboratories", coauthored by D. Ursutiu, D. Iordache, P.A. Cotfas, D.T. Cotfas and C. Samoila. This paper presents a solution for implementing remote labs by using web technologies that are platform independent from the client point of view. Such a lab implementation exemplification is materialized by remote controlling the NI-ELVIS platform.

We hope that you enjoyed this special issue and we look forward to your participation in future issues of REV (www.rev-conference.org). 